\title{
Influence of the cross-section geometry of a cylindrical solid submerged in an acoustic medium on wave propagation
}

\author{
Andreia Pereira, António Tadeu*, Julieta António \\ Department of Civil Engineering, University of Coimbra, Polo II-Pinhal de Marrocos, 3030-290 Coimbra, Portugal
}

Received 12 February 2001; received in revised form 28 May 2001; accepted 14 August 2001

\begin{abstract}
This paper studies wave propagation in the vicinity of a cylindrical solid formation submerged in an acoustic medium generated by point blast loads placed outside the inclusion. The full 3D solution is obtained first in the frequency domain as a discrete summation of responses for 2D problems defined by a spatial Fourier transform. Each 2D solution is computed using the Boundary Element Method, which makes use of two-and-a-half-dimensional Green's functions. This model is implemented to obtain Fourier spectra responses which make it possible to identify the behavior of both the axisymmetric and non-axisymmetric guided wave modes, when the cross-section of the elastic inclusion changes from circular to smooth oval.

When the cylindrical elastic inclusion is submerged in a fluid, thus allowing a dilatational wave velocity greater than the shear wave velocity of the elastic medium (slow formation), our computations show a progressively slower flexural wave and the increased importance of a second flexural mode as the ovality of the inclusion becomes more pronounced. The waves associated with the screw mode become less important as the ovality ratio of the inclusion increases.

When the formation is fast (the shear wave velocity of the cylindrical solid inclusion is faster than the pressure wave velocity of the fluid), the responses again indicate a progressively slower flexural wave, as the inclusion becomes more oval. The results computed at the receivers placed on the axis appear to be weakly affected by the ovality ratio of the inclusion. () 2002 Elsevier Science B.V. All rights reserved.
\end{abstract}

\section{Introduction}

The propagation of acoustic waves in the vicinity of elastic inclusions or formations has been studied for a number of years. Different approaches have been developed to try to reproduce the phenomena involved.

Most of the work related to this field relies on research by geophysicists and seismologists into the development of seismic prospecting techniques (acoustic logging, vertical profiling and cross-hole surveying) to predict subterranean characteristics, and by ocean and aeronautical researchers to study the interaction of the fluid with shell-shaped and solid structures.

Biot [1] did the first theoretical work on the dispersion equation for guided waves, and their phase and group velocities, along a borehole. Since then, a large number of researchers have addressed the problem of wave propagation along fluid-filled boreholes subjected to plane waves and point sources, placed on and away from the axis of the borehole.

\footnotetext{
* Corresponding author. Tel.: +351-239-797-204; fax: +351-239-797-190.

E-mail address: tadeu@dec.uc.pt (A. Tadeu).
} 
Recently, several researchers have investigated the propagation of waves along fluid-filled boreholes from sources aligned with the borehole axis, in a context of acoustic logging. Schoenberg [2] developed explicit formulas, valid for low frequencies, for the incidence of compressional or shear plane waves on the fluid borehole. Lovell and Hornby [3] later provided expressions valid for all frequencies and incidence angles.

Numerical methods have been proposed for studying how the formation characteristics of the material, the type of fluid in the borehole and the source influence wave amplitude and attenuation [4-6]. Different data processing schemes have been suggested by Tezuka et al. [7] and Herman et al. [8]. The estimation of the mechanical properties of the formation has been addressed by Cheng et al. [9]. This work was later extended to study the effect of the state of fracturing and the presence of damaged zones around the borehole [10]. The impact of transversely isotropic formations was also investigated [11,12]. Finite element methods have been proposed to compute borehole normal modes and waveforms in the anisotropic formation [13,14]. Later, Dong and Toksöz [15] computed the radiation patterns of downhole seismic sources inside a fluid-filled borehole driven in a transversely isotropic formation. Randall [16] calculated dispersion curves for the modes of non-circular fluid-filled boreholes in homogeneous elastic formations, using a boundary integral formulation. Results for the propagation modes of several borehole shapes in both fast and slow formations were given.

Kurkjian et al. [17] proposed a numerical technique for solving downhole seismic data in cross-well configurations that took into account the generation of tube waves in the borehole source, adopting a procedure for the transmission from the source well and finally computing the receivers responses by applying the White's quasi-static approximation. Recently, Peng et al. [18] used both the borehole coupling theory and the global matrix formulation for calculating synthetic seismograms in a layered medium, avoiding discretization along the borehole.

The finite difference method was applied by Randall [19] to study monopole and dipole acoustic logs. Later, Cheng et al. [20] used a true 3D finite difference method, using a parallel computing scheme, to compute the time domain wave propagation response for a borehole in an anisotropic formation.

In the context of the presence of shell and elastic inclusions submerged in a fluid medium, the literature shows that, since Pochammer [21], who derived the dispersion equation for waves propagating in circular rods, a number of researchers have contributed to this area of research. The interaction of solid rods with fluid media, in particular, has been focused on. Longitudinal waves in homogeneous anisotropic cylindrical circular bars immersed in a fluid medium have been computed numerically by Dayal [22], using cylindrical equations to find the dispersion curves and the attenuation caused by the energy leaked into the fluid. Results are presented for rods made from five different materials. Van den Abeele and Leroy [23] computed bounded beam reflection and transmission at layered media. They extended the method of Claeys and Leroy [24] to a more general approximation, using both positive and negative decaying heterogeneous waves. Furuyama and Inoue [25] computed the propagation of weakly nonlinear waves radiated by a rigid circular cylinder oscillating harmonically in a direction perpendicular to its axis, submerged in a perfect gas.

Stanton [26] obtained approximate analytical expressions to compute the scattering of sound by rough, elongated elastic objects. Ramakrishna and Stepanishen [27] presented a numerical formulation for defining the acoustic harmonic pressure fields, both scattered and total, that arise when rigid infinitely long cylinders, with cross-sections which are symmetric about an axis, are illuminated by $2 \mathrm{D}$ incident pressure waves. This formulation is based on the determination of monopole and dipole source distributions within the surface via a least-mean-square error scheme, by matching the normal velocity field from the internal sources at the surface of the rigid cylinder with the normal surface velocity produced by the incident wave field.

Stanton et al. [28] subsequently used the distorted wave 'Born approximation' to predict weak scattering by spheres and finite-length cylinders, as produced by zooplankton. Their technique is restricted to weakly scattering materials, in that the density and sound velocity of the inclusion material must be very similar to that of the surrounding medium. Before this work, the zooplankton had been modeled mathematically, almost exclusively as spheres, as summarized in Holliday and Pieper [29].

The resonance scattering theory, referenced as the resonance formalism of the nuclear reaction theory, has been used to solve acoustic and elastic wave scattering problems. In this technique, the partial wave contained in the total scattering amplitude is decomposed into two parts: one is known as the background, which is smooth and 
regular, and the other is the set of modal resonances of the scatterer. Joo et al. [30] used this technique to deduce expressions to obtain the inherent background coefficients of multilayered cylindrical circular structures subjected to plane waves.

In a context of lithotripsy developments, Dahake and Gracewski [31,32] analyzed the wave field in acoustic and elastic media using regular and irregular geometries generated by a 2D radially diverging pressure pulse source, using time domain finite differences techniques. The responses are first computed for solid cylinders with rectangular or circular cross-sections, subjected to a line source placed in a surrounding fluid. They then studied the internal stress wave field generated within submerged solids of various shapes, subjected to a radially diverging source placed in the surrounding fluid.

Niklasson and Subhendu [33] computed the scattering by a circular cylinder in a transversely isotropic elastic medium. The problem is solved using separation of variables in cylindrical coordinates. Sinclair and coworkers [34] presented a normal mode expansion solution to compute the waves scattered by a transversely isotropic cylinder embedded in a solid elastic medium, when subjected to a plane wave incident at an arbitrary angle. The geometry of their model approaches that of a composite material featuring a reinforced fiber encased in an elastic matrix.

Most work has focused on the plane harmonic scattering of cylindrical circular inclusions, using different numerical approaches. In our work the full wave propagation in the vicinity of an elastic formation submerged in an unbounded fluid medium, generated by a point pressure source placed inside the fluid, is studied. Both frequency and time solutions are provided to study the effects of cross-section geometry variation, on the wave scattering. The circular cross-section responses are used as a reference for the results obtained when the geometry changes to accommodate ovals with different ovality ratios, while keeping the perimeter of the former circular inclusion. This work tries to improve understanding of how guided waves, generated by loads in the vicinity of solid structures submerged in a fluid medium, propagate. It aims to help define future construction procedures which will prevent the propagation of these waves along solid structures. The first phase of this research is presented here; it considers a long solid inclusion (a rod) submerged in a water medium. The results of this work are also applicable to the development of non-destructive techniques.

The boundary element method (BEM) is the technique used to solve the present problem, because it is probably one of the most suitable techniques for modeling wave scattering when the far field radiation conditions need to be satisfied. The BEM has already been applied by Sarkissian [35] to compute the radiation of scattering from multiple cylinders submerged in a fluid medium, assuming the existence of prescribed normal velocity distributions on the cylinders surfaces. Numerical results are presented for various two-or-three-cylinder configurations, where a plane harmonic wave is incident from various angles. Bouchon [36] also used the BEM to solve the case of an infinite open borehole in layered isotropic media. Later, Dong et al. [37] included the effect of casing and cement in a layered, transversely isotropic formation, using an indirect BEM formulation.

The geometry of our problem can be referred as a two-and-a-half-dimensional geometry, because the medium is assumed to be $2 \mathrm{D}$, and the source excited is $3 \mathrm{D}$. As the geometry of the problem does not vary along the $z$-direction (with $z$ being the longitudinal axis of the solid formation), the solution can be obtained by solving a sequence of $2 \mathrm{D}$ problems for a varying wavenumber along the $z$-direction, $k_{z}$. This procedure is possible after a prior spatial Fourier transformation along the $z$-direction. The required summation of 2D solutions is rendered discrete by assuming the existence of an infinite number of virtual sources spaced at equal intervals along the $z$-axis, at a certain minimum distance from each other in order to avoid spatial contamination. The effects of the neighboring fictitious sources are minimized by using complex frequencies for the analyses.

This model is implemented and used to understand wave propagation variation when the cross-section of the elastic inclusion changes. The circular cross-section responses are used as a reference for the results obtained when the geometry changes to accommodate oval cross-sections with different ovality ratios, but retaining the perimeter of the former circular inclusion.

This model is used to identify the behavior of both the axisymmetric and non-axisymmetric normal modes. The variables used in this study are: type of formation, defined by the relation between the shear velocity of the 
solid medium and the dilatational wave velocity of the fluid; the position of the receivers, and the shape of the cross-section.

Next, the $3 \mathrm{D}$ problem is described and the $\mathrm{BEM}$ is formulated in the frequency domain. Then, the numerical validation of the results is presented, using a circular cylindrical solid formation model, for which analytical expressions exist. The responses in the time domain are subsequently obtained. The method is then implemented to study the wave propagation around elastic inclusions with different cross-sections. Finally, conclusions are drawn.

\section{Problem formulation}

Consider a cylindrical irregular solid formation of infinite extent, submerged in a spatially uniform fluid medium (Fig. 1), subjected to a harmonic dilatational pressure source at position $\mathrm{O}$, oscillating with a frequency $\omega$,

$$
p_{\text {inc }}=\frac{A \mathrm{e}^{\mathrm{i}(\omega / \alpha)\left(\alpha t-\sqrt{x^{2}+y^{2}+z^{2}}\right)}}{\sqrt{x^{2}+y^{2}+z^{2}}}
$$

in which the subscript 'inc' denotes the incident field, $A$ the wave amplitude, $\alpha$ the compressional wave velocity of the medium and $\mathrm{i}=\sqrt{-1}$.

Fourier-transforming equation (1) in the $z$-direction, and defining the effective wavenumbers $k_{\alpha}=\sqrt{\left(\omega^{2} / \alpha^{2}\right)-k_{z}^{2}}$, one obtains

$$
\hat{p}_{\text {inc }}\left(\omega, x, y, k_{z}\right)=-\frac{\mathrm{i} A}{2} H_{0}^{(2)}\left(k_{\alpha} \sqrt{x^{2}+y^{2}}\right)
$$

in which the $H_{n}^{(2)}(\ldots)$ are second Hankel functions of order $n$.

This procedure allows the solution to be obtained as a summation of 2D solutions. Considering an infinite number of virtual pressure sources at equal spaces, $L$, along the $z$-direction, the solution can be computed as a discrete summation of solutions for varying wavenumbers $k_{z m}=(2 \pi / L) m$. In these calculations, $L$ must be sufficiently large to avoid spatial contamination [38].

The 3D field generated when an elastic formation, submerged in an infinite fluid medium, is illuminated by a spatially sinusoidal harmonic line load, defined by Eq. (2), is computed here by utilizing the BEM. The numerical formulation of this method, applied to wave propagation, has been widely studied [39-41]. It is known that the computation of the scattered wave field in the frequency domain requires the evaluation of the following integrals,

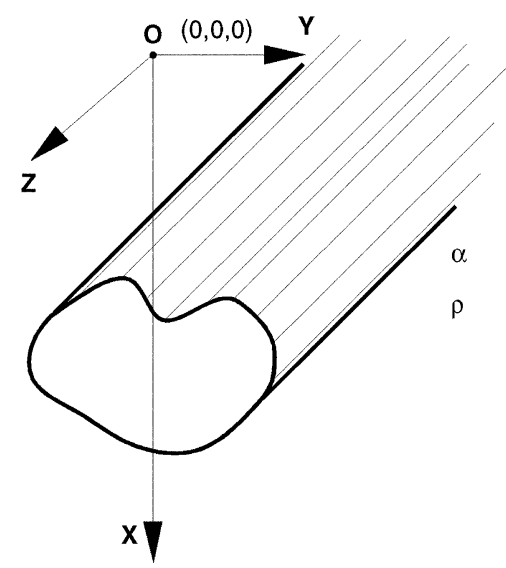

Fig. 1. Geometry of the problem. 
at each element of the discretized inclusion boundary:

$$
\begin{array}{lll}
H_{i j}^{(\mathrm{s}) k l}=\int_{C_{l}} H_{i j}^{(\mathrm{s})}\left(x_{k}, x_{l}, n_{l}\right) \mathrm{d} C_{l}, & i, j=1,2,3, & H_{\mathrm{f} 1}^{(\mathrm{f}) k l}=\int_{C_{l}} H_{\mathrm{f} 1}^{(\mathrm{f})}\left(x_{k}, x_{l}, n_{l}\right) \mathrm{d} C_{l}, \\
G_{i j}^{(\mathrm{s}) k l}=\int_{C_{l}} G_{i j}^{(\mathrm{s})}\left(x_{k}, x_{l}\right) \mathrm{d} C_{l}, & i=1,2,3, j=1, \quad G_{\mathrm{f} 1}^{(\mathrm{f}) k l}=\int_{C_{l}} G_{\mathrm{f} 1}^{(\mathrm{f})}\left(x_{k}, x_{l}\right) \mathrm{d} C_{l}
\end{array}
$$

In these equations, $H_{i j}^{(\mathrm{s})}\left(x_{k}, x_{l}, n_{l}\right)$ and $G_{i j}^{(\mathrm{s})}\left(x_{k}, x_{l}\right)$ are, respectively, the Green's tensor for traction and displacement components in the elastic medium, at the point $x_{l}$ in direction $j$ caused by a concentrated load acting at the source point $x_{k}$ in direction $i ; H_{\mathrm{f} 1}^{(\mathrm{f})}\left(x_{k}, x_{l}, n_{l}\right)$ are the components of the Green's tensor for pressure in the fluid medium, at the point $x_{l}$, caused by a pressure load acting at the source point $x_{k} ; G_{\mathrm{f} 1}^{(\mathrm{f})}\left(x_{k}, x_{l}\right)$ are the components of the Green's tensor for displacement in the fluid medium, at the point $x_{l}$, in the normal direction, caused by a pressure load acting at the source point $x_{k} ; n_{l}$ is the unit outward normal for the $l$ th boundary segment $C_{l}$; the subscripts $i, j=1,2,3$ denote the normal, tangential and $z$-directions, respectively. Tadeu and Kausel [42] presents the two-and-a-half-dimensional fundamental solutions (Green's functions) in the Cartesian coordinates required for the elastic and fluid media. Expressions for the tensions may be obtained from the Green's functions by taking partial derivatives to deduce the strains and then applying Hooke's law to obtain the stresses.

The integral equations (3) are then manipulated in order to verify the boundary conditions between the two media, i.e. the continuity of normal displacements and stresses, and null tangential stresses at the interface between the solid and the fluid. Solving the resulting system makes it possible to obtain nodal solid displacements and fluid pressures.

The integrations in Eq. (3) are computed in closed form when the element to be integrated is the loaded element, and by means of Gaussian quadrature when the element to be integrated is not the loaded element.

\section{Validation of the BEM algorithm}

The BEM algorithm used in this work was implemented and validated by applying it to a cylindrical circular solid inclusion $\left(\alpha=2630 \mathrm{~m} / \mathrm{s}, \beta=1416 \mathrm{~m} / \mathrm{s}\right.$ and $\left.\rho=2250 \mathrm{~kg} / \mathrm{m}^{3}\right)$ submerged in an inviscid fluid medium $\left(\alpha_{\mathrm{f}}=1500 \mathrm{~m} / \mathrm{s}\right.$ and $\left.\rho_{\mathrm{f}}=1000 \mathrm{~kg} / \mathrm{m}^{3}\right)$, subjected to a dilatational harmonic line load applied at point $\mathrm{O}$, with $k_{z}=1.0 \mathrm{rad} / \mathrm{m}$, placed as shown in Fig. 2, for which the solution is known in closed form (described in Appendix A).

The response is calculated at a receiver placed inside the elastic formation. Computations are performed in the frequency range $25.0-1600.0 \mathrm{~Hz}$. Fig. 2 displays the normalized scattered field along the $z$-direction, calculated when the inclusion is modeled with 150 boundary elements. The normalization procedure used the maximum amplitude of the response, within the frequency range computed, for reference purposes. In this figure, the solid line represents the analytical solution, and the marks illustrate the BEM solution. Analysis of the results confirms a good agreement between the two solutions.

\section{Results in the time domain}

Responses (displacements and pressures) in the spatial-temporal domain are obtained using a fast inverse Fourier transform in the frequency and wavenumber domain. The source used to calculate the temporal solution is modeled as a Ricker wavelet

$$
u(\tau)=A\left(1-2 \tau^{2}\right) \mathrm{e}^{-\tau^{2}}
$$

where $A$ is the amplitude, $\tau=\left(t-t_{\mathrm{s}}\right) / t_{0}$ and $t$ denotes time, $t_{\mathrm{s}}$ the time when the maximum occurs, while $\pi t_{0}$ the characteristic (dominant) period of the wavelet. Its Fourier transform is

$$
U(\omega)=A\left[2 \sqrt{\pi} t_{0} \mathrm{e}^{-\mathrm{i} \omega t_{\mathrm{s}}}\right] \Omega^{2} \mathrm{e}^{-\Omega^{2}}
$$

in which $\Omega=\frac{1}{2} \omega t_{0}$. 


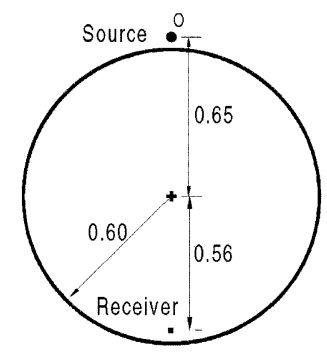

Fluid: $\alpha_{f}=1500 \mathrm{~m} / \mathrm{s}$

$$
\rho_{f}=1000 \mathrm{~kg} / \mathrm{m}^{3}
$$

Solid: $\alpha=2630 \mathrm{~m} / \mathrm{s}$

$$
\begin{aligned}
& \beta=1416 \mathrm{~m} / \mathrm{s} \\
& \rho=2250 \mathrm{~kg} / \mathrm{m}^{3}
\end{aligned}
$$

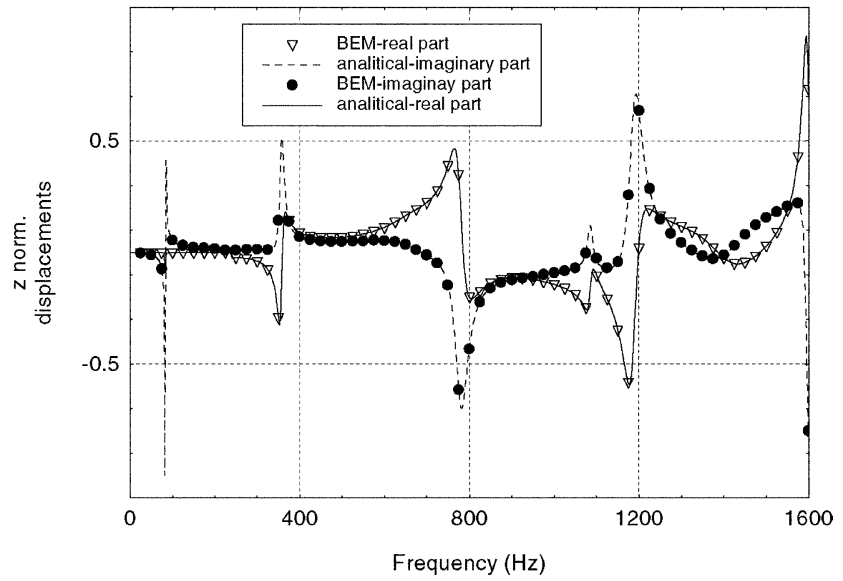

Fig. 2. Validation of the BEM.

This wavelet form has been chosen because it decays rapidly, both in time and frequency, reducing computational effort and allowing easier interpretation of the computed time series and synthetic waveforms.

As stated before, the Fourier transformations are obtained by discrete summations over wavenumbers and frequencies. Mathematically, this is achieved by adding periodic sources at spatial intervals $L=2 \pi / \Delta k_{z}$ (in the $z$-axis), and temporal intervals $T=2 \pi / \Delta \omega$, with $\Delta k_{z}$, and $\Delta \omega$ being the wavenumber and frequency steps, respectively [38]. The spatial separation, $L$, must be large enough to guarantee that the response of the fictitious sources occurs at times later than $T$, and therefore avoids contamination. Complex frequencies, with a small imaginary part of the form, $\omega_{\mathrm{c}}=\omega-\mathrm{i} \eta$ (with $\eta=0.7 \Delta \omega$ ), can also be used to improve the accuracy of the response by introducing a significant attenuation, or virtual elimination, of the periodic sources. In the time domain, this shift is later taken into account by applying an exponential window $\mathrm{e}^{\eta t}$ to the response [43].

\section{Numerical applications}

The 3D boundary element program was implemented and applied to the numerical analysis of a cylindrical solid inclusion submerged inside a fluid medium when illuminated by a point pressure load. Different cross-sections, with a constant perimeter $(1.2 \pi \mathrm{m})$, were simulated. In this work, only a circle with radius of $R=0.6 \mathrm{~m}$ and a thin oval, with an ovality ratio of $\varepsilon=1.68 / 0.62=2.7$ are used to illustrate the main findings. The wave field generated is computed at receivers located as shown in Fig. 3, on five planes, equally spaced $(6.0 \mathrm{~m})$ along the $z$-direction.

The influence of the ratio between the shear velocity of the solid medium and the dilatational wave velocity of the fluid on the wave propagation is another parameter analyzed. This work examines two different formations (see Table 1), identical to the ones used by Ellefsen [13] in his studies on the determination of the phase and group velocities of a fluid-filled circular borehole.

The calculations were performed in the frequency range from 25 to $1600 \mathrm{~Hz}$ with a frequency increment of $25 \mathrm{~Hz}$. The total time taken for the analysis is given by $T=\frac{1}{25}=40 \mathrm{~ms}$. Therefore, the spatial period chosen for the calculations cannot be less than $L=2 T \alpha$, which gives $L=2 T \alpha=336 \mathrm{~m}$ and $L=2 T \alpha=212 \mathrm{~m}$ in the presence of a fast formation and a slow formation, respectively. The imaginary part of the angular frequency is set 

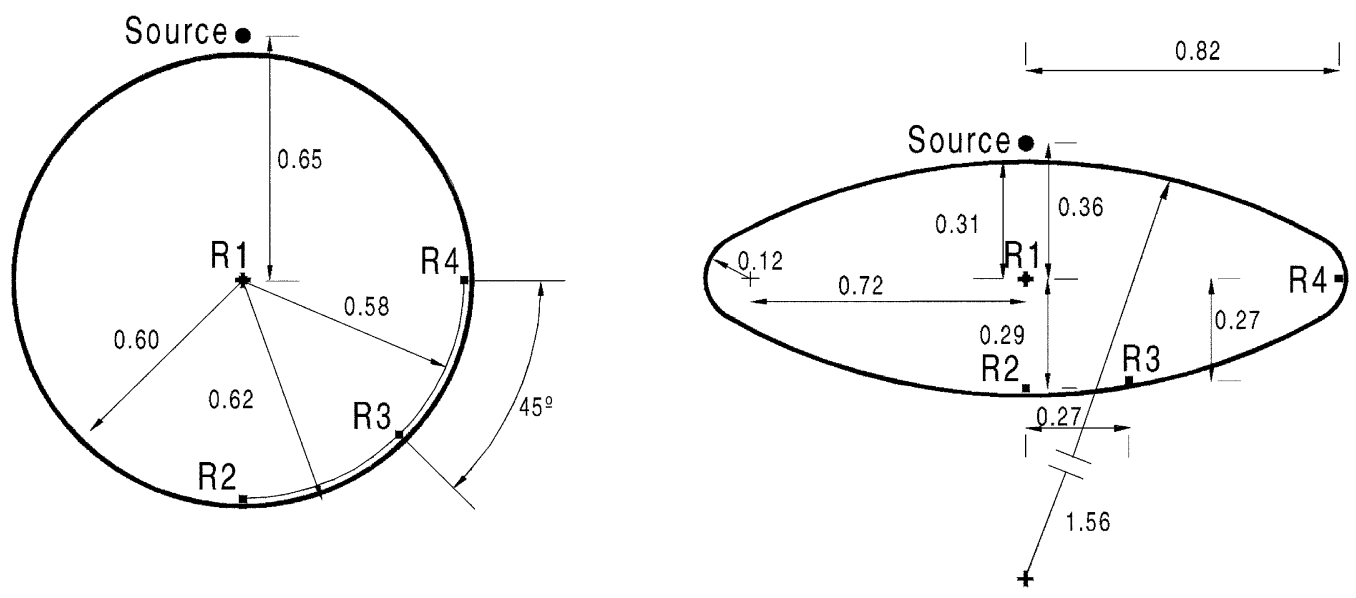

Fig. 3. Cross-sections of the solid formation.

to $\eta=0.7 \Delta \omega$, attenuating the wraparound by a factor of $\mathrm{e}^{0.7 \Delta \omega T}=81$ (i.e. $38 \mathrm{~dB}$ ). The source is a Ricker wavelet pulse with a characteristic frequency of $500 \mathrm{~Hz}$. The results were computed for $x, y$ and $z$ component displacements. Given the symmetry of the problem, the $x$ displacements register null value at receivers placed on the $y$ axis $(x=0)$. The main features of the computed wave field are illustrated using the $z$ displacements in the solid formation, which would be similar to the results obtained along the other component displacements. Each time plot is normalized with respect to the maximum time response obtained at receiver 1 for a distance in the $z$-direction of $6 \mathrm{~m}$, when the inclusion is circular.

The cross-sections were modeled with boundary elements, the number of which varied with the excitation frequency of the harmonic load. The ratio between the wavelength of the incident field and the length of the boundary element used was 12 . The minimum number of boundary elements considered for any of the analyses was never less than 40 .

The various waves propagating in the inclusion and its vicinity include non-dispersive (body waves), namely the dilatational $\left(P_{\mathrm{s}}\right)$ and shear $\left(S_{\mathrm{s}}\right)$ waves in the solid medium, and pressure $\left(P_{\mathrm{f}}\right)$ waves in the fluid medium. In addition, there are guided waves propagating along the surface of the solid inclusion, which correspond to axisymmetric modes and modes with azimuth variation. Some of these modes are excited only if the source excitation frequency exceeds a certain value. This value is termed the cutoff frequency.

The dispersion characteristics of the normal modes can be obtained by solving an eigenvalue problem in the absence of an incident field. The associated eigenvalues $k_{z}$, lead in turn to the phase and group velocities of the normal modes. While an infinite (but countable) number of modes exist, only those with a low modal order contribute significantly to the response.

To better illustrate the behavior of the guided waves, the Fourier spectra for waves traveling with velocities of between 700.0 and $1600.0 \mathrm{~m} / \mathrm{s}$ are displayed. Notice that these plots do not correspond to the required integration,

Table 1

Mechanical properties of the two formations

\begin{tabular}{lll}
\hline & Fast formation & Slow formation \\
\hline$\alpha(\mathrm{m} / \mathrm{s})$ & 4208 & 2630 \\
$\beta(\mathrm{m} / \mathrm{s})$ & 2656 & 1416 \\
$\rho\left(\mathrm{kg} / \mathrm{m}^{3}\right)$ & 2140 & 2250 \\
$\alpha_{\mathrm{f}}(\mathrm{m} / \mathrm{s})$ & 1500 & 1500 \\
$\rho_{\mathrm{f}}\left(\mathrm{kg} / \mathrm{m}^{3}\right)$ & 1000 & 1000 \\
\hline
\end{tabular}



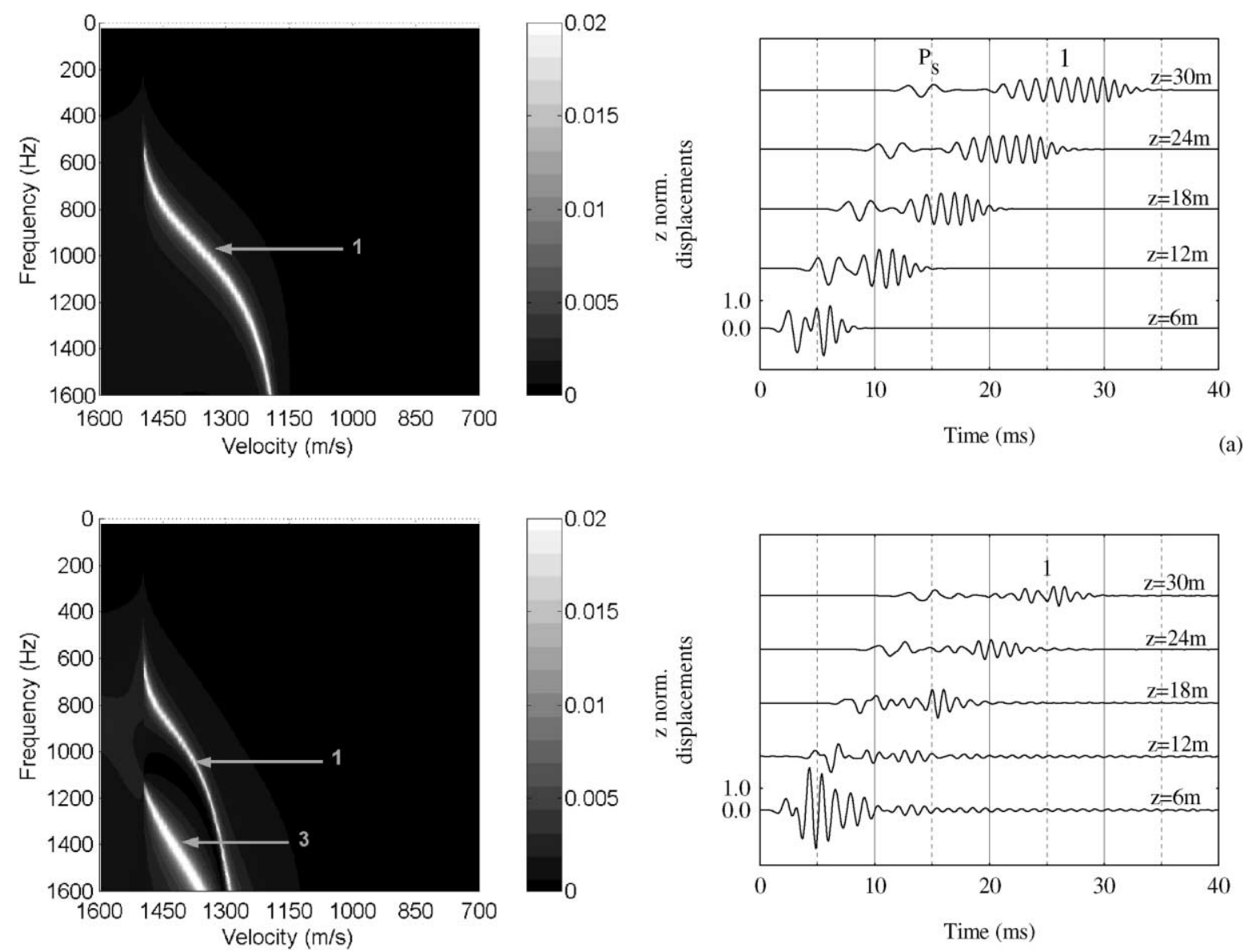

1- Axisymmetric, 3- Screw

(b)

Fig. 4. Frequency spectra and time responses at receiver 1, when the formation is slow: (a) circular inclusion; (b) thin oval inclusion.

where the increment of $\Delta k_{z}$ was set to be $2 \pi / L$. Each plot is again normalized with respect to the maximum response obtained at receiver 1 , when the inclusion is circular. The Fourier spectra plots use a gray scale where lighter and darker shades are ascribed to higher and lower amplitude values, respectively.

\subsection{Slow formation}

Figs. 4-6 give the responses computed when the solid formation is driven in a slow formation. Fig. 4 displays the responses at receiver 1 placed on the axis.

A source placed off the axis excites both axisymmetric and non-axisymmetric modes, but only the former contribute to the pressure on the axis when the inclusion is circular. In the case of the circular inclusion therefore, only the body waves and the waves from the axisymmetric mode are observed. The time plots reveal the existence of the dilatational $P_{\mathrm{S}}$ waves and the pulses associated with the axisymmetric mode. As we move from the circular to the thin oval $\varepsilon=2.7$, these waves exhibit a faster velocity and a lower amplitude, as the spectra plots illustrate. The time plots confirm this phenomenon by eliminating the later arrivals and diminishing the amplitude of the corresponding pulses. Furthermore, as the ovality ratio increases, our results indicate the existence of a second mode, with velocities 

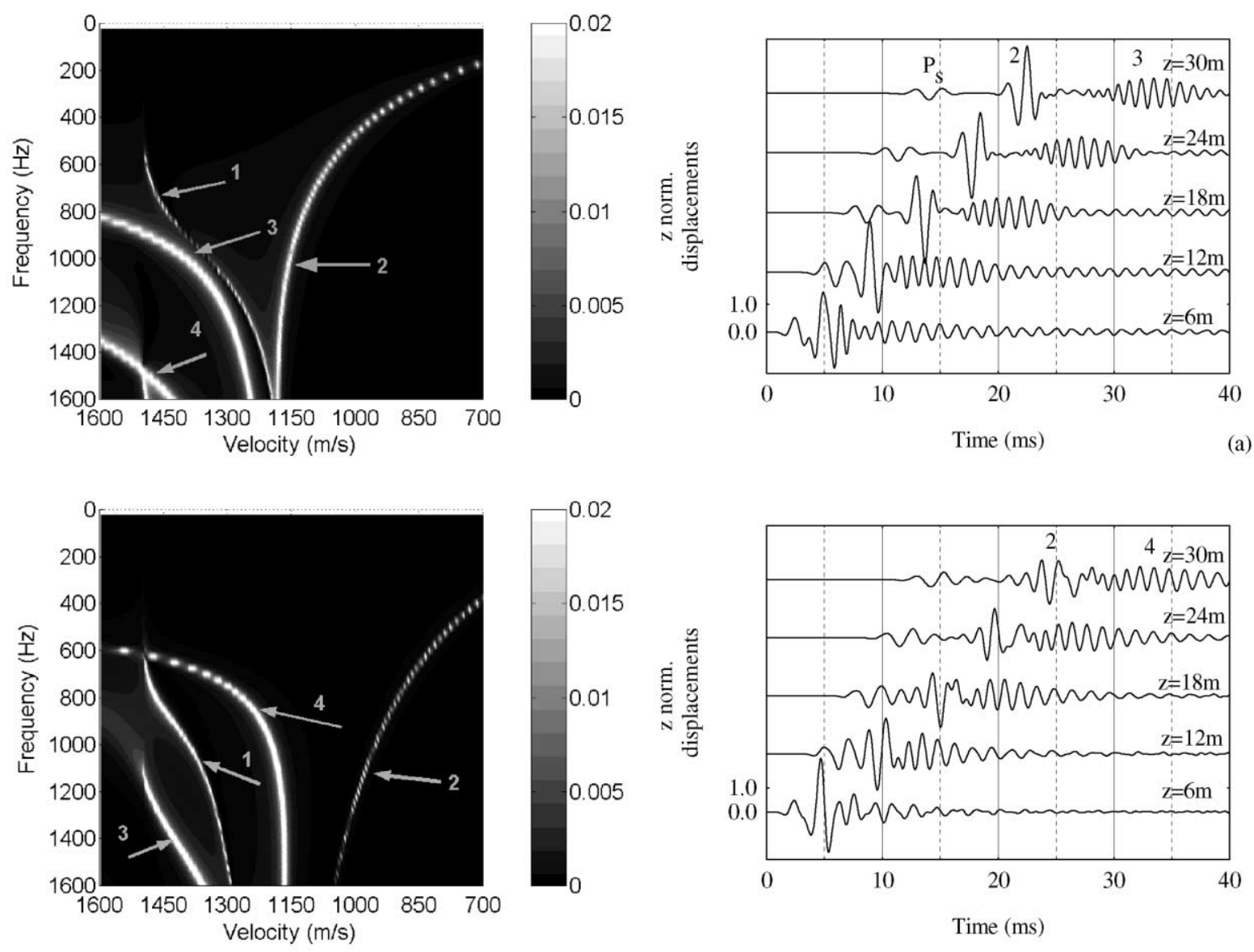

1- Axisymmetric, 2- Flexural, 3- Screw, 4 - Second Flexural

(b)

Fig. 5. Frequency spectra and time responses at receiver 2, when the formation is slow: (a) circular inclusion; (b) thin oval inclusion.

similar to the screw mode. This mode makes a very small contribution to the time responses, given the frequency of the pulse excited $(500 \mathrm{~Hz})$, which is much lower than the cutoff frequency of these waves $(\approx 0.8 \mathrm{kHz})$.

Fig. 5 illustrates the response close to the wall of the borehole, at receiver 2, placed in the same azimuth direction as the source. The responses at this receiver include contributions from the flexural and screw waves, in addition to the waves corresponding to the axisymmetric modes.

Analysis of the responses shows that their spectral representation allows the different wave types to be distinguished visually. It indicates that the group velocity of screw waves is lower than that for those associated with the axisymmetric mode, which in turn is lower than for the flexural waves. Notice that the group velocity can be obtained by the relationship $\mathrm{d} \omega / \mathrm{d} k_{z}$. The waves associated with the axisymmetric mode are weaker than those found at receiver 1, placed on the axis. As we proceed from the circular inclusion to the thin oval, we notice that the flexural waves become slower. The time plots show this in the progressive delay of the flexural arrivals. Our results further indicate that a second flexural mode starts to be important as the cross-section becomes more oval, exhibiting lower cutoff frequencies and lower group velocities. The time plots of the thin oval demonstrate this behavior by introducing later time arrivals with higher amplitudes. Meanwhile, the screw waves lose importance as we move from the circular to the thin oval, because they appear later in the frequency domain. 

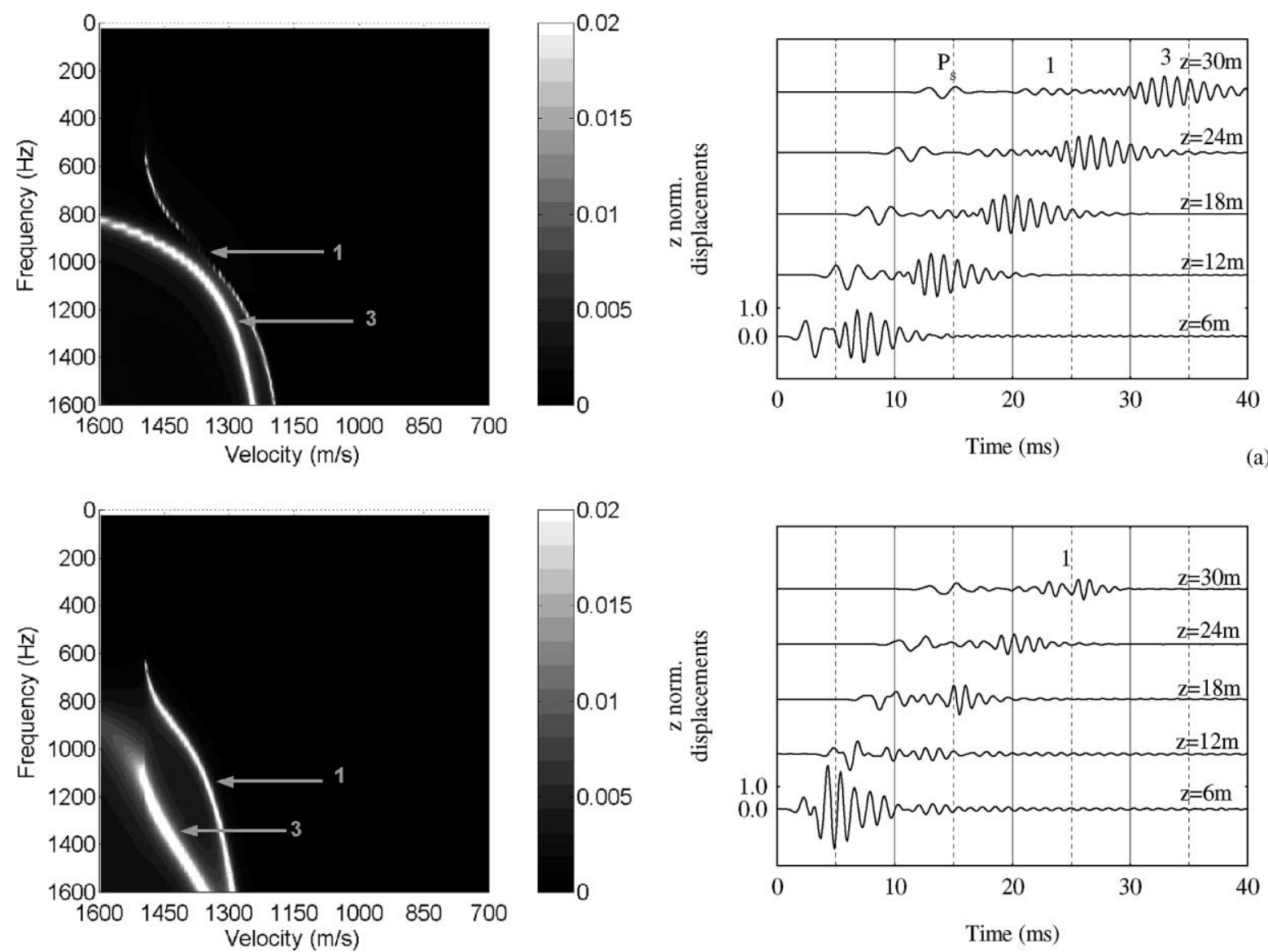

1- Axisymmetric, 3- Screw

(b)

Fig. 6. Frequency spectra and time responses at receiver 4, when the formation is slow: (a) circular inclusion; (b) thin oval inclusion.

As we move to receiver 3 , located along a plane at $45^{\circ}$ (not displayed), the spectra plots do not reveal the existence of the screw mode when the cross-section is circular. The time plots confirm this behavior by erasing the later pulses registered at receiver 2 . When the cross-section changes from the circular to thin oval, this mode becomes important, although its amplitude is lower than at receiver 2. The contribution of the flexural mode is lower than it was for receiver 2 , because the receiver is nearer to the neutral axis.

The signatures at receiver 4 are not affected by flexural waves (see Fig. 6), because the flexural mode has zero amplitude on the neutral axis. This axis is the horizontal plane through the axis that is perpendicular to the line connecting the center with the source. Hence, after the passage of the $P_{\mathrm{s}}$ waves, the signatures on the neutral axis experience a substantial drop in amplitude, before the arrival of the screw waves. Notice that this drop is not observed at receiver 2, located away from the neutral axis (see Fig. 5). The screw waves manifest behavior similar to that described for receiver 2 . When the oval is thin, the relative importance of the waves associated with the axisymmetric mode increases.

The responses obtained for the receivers located within the fluid media behave in a similar fashion (not shown). The time plots show the existence of the pressure $\left(P_{\mathrm{f}}\right)$ body waves in the fluid media, in addition to the pulses associated with the surface modes (not shown). 

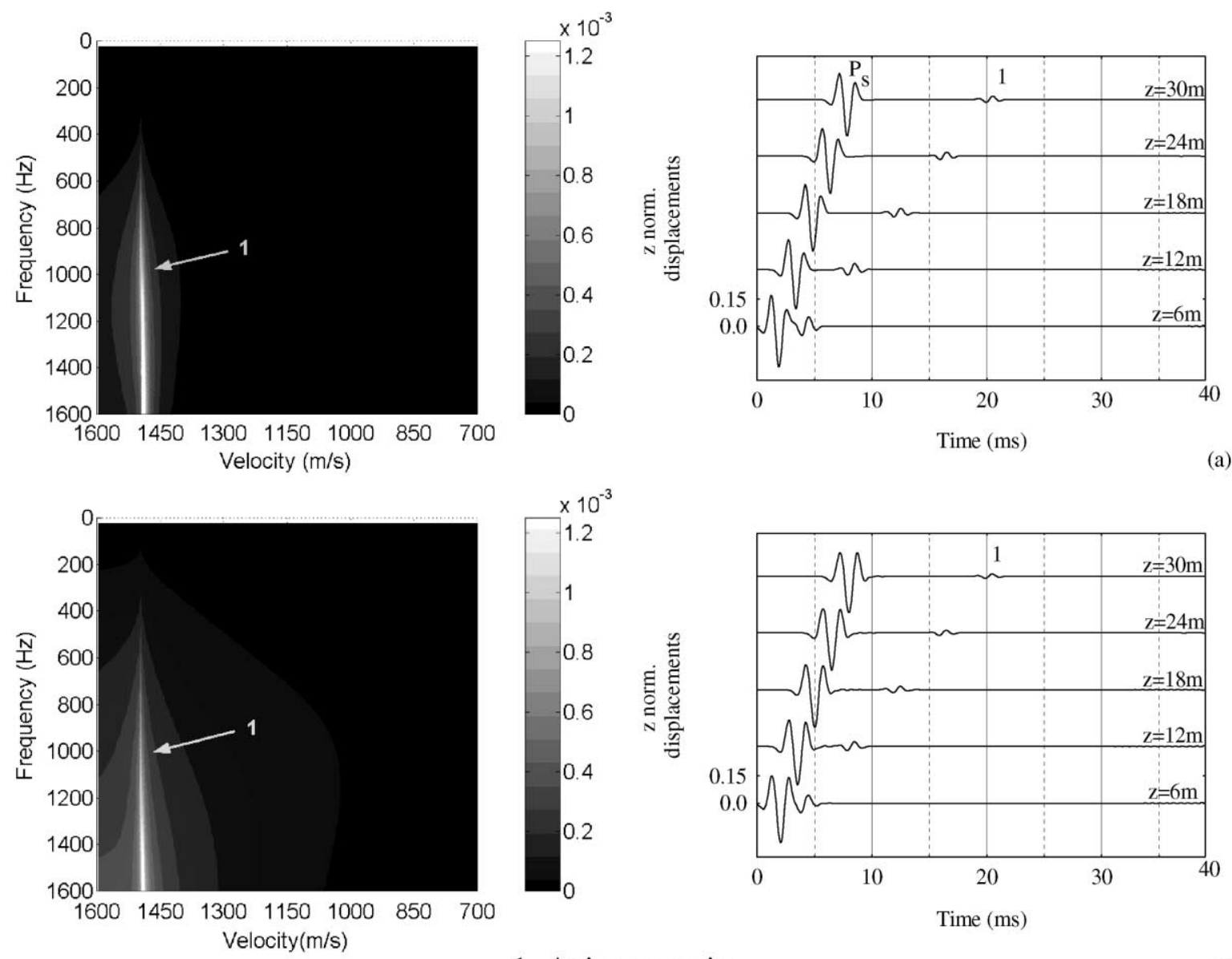

1- Axisymmetric

(b)

Fig. 7. Frequency spectra and time responses at receiver 1, when the formation is fast: (a) circular inclusion; (b) thin oval inclusion.

\subsection{Fast formation}

Figs. 7 and 8 display the responses obtained at receivers 1 and 2 when the borehole is driven in fast formation. When the shear wave velocity is faster than the dilatational fluid velocity, only the lowest order radial modes exist, since no critical dilatational refraction can occur.

At receiver 1 (see Fig. 7), placed on the borehole axis, the axisymmetric mode alone is excited for all cross-sections studied. Thus, the time plots only show the presence of two pulses, the dilatational body $\left(P_{\mathrm{s}}\right)$ wave and the pulse associated with the axisymmetric mode. The spectra analysis also indicates that responses are not highly dispersive. The responses for all borehole sections are similar.

The responses computed at the receiver 2 (see Fig. 8) show the contribution of the flexural and axisymmetric modes. The presence of the screw mode is not detected in these plots because of the frequency excited $(500 \mathrm{~Hz})$, which is much lower than the cutoff frequency of these waves. As in the slow formation, the group velocities of the flexural waves become slower when the cross-section changes from the circular, to the thin oval, as the time plots confirm. However, as the frequency increases, the flexural wave velocity approaches the velocity of the compressional fluid wave velocity, in all cross-sections. 

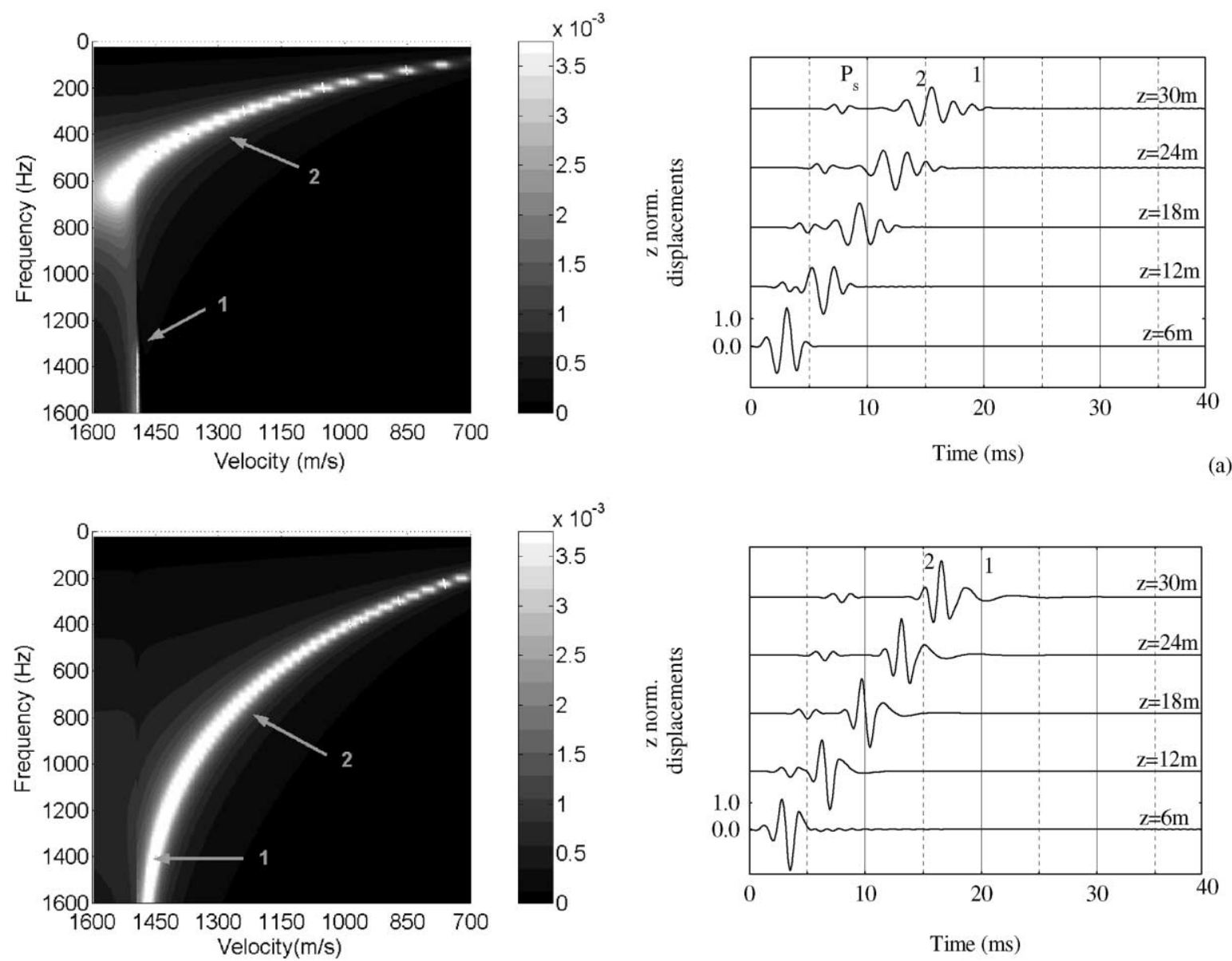

1- Axisymmetric, 2- Flexural

(b)

Fig. 8. Frequency spectra and time responses at receiver 2, when the formation is fast: (a) circular inclusion; (b) thin oval inclusion.

It should be noted that the results presented in this paper consider only smooth cross-sectional geometries. The presence of a cross-section with irregularities such as the existence of sharp corners would introduce additional wave phenomena complexity.

\section{Conclusions}

A boundary element formulation was implemented to evaluate the 3D wavefield generated by a pressure point source striking an elastic inclusion (in a fast and slow formation) submerged in a fluid medium. This model was used to assess the influence of the receiver position on the propagation of both the axisymmetric and non-axisymmetric wave modes when different inclusion cross-sections were used, namely a circular and a thin oval. Time signatures were computed using complex frequencies. Their Fourier spectral representations were presented, allowing a better separation of the different wave modes. 
When the cylindrical elastic inclusion is submerged in a fluid, where the shear velocity of the solid medium is lower than the dilatational wave velocity of the fluid (slow formation), the results indicate a progressively slower flexural wave and the increased importance of a second flexural mode, which appears earlier in the frequency domain, as the ovality of the inclusion increases. Meanwhile, the waves associated with the screw mode lose importance in the time domain as the ovality ratio increases, because they arrive later in the frequency domain. The waves related to the axisymmetric mode appear more relevant at the receiver placed on the axis. In addition, they become faster as the ovality ratio grows. The results computed for receivers placed in the fluid media, close to the inclusion surface, exhibit features similar to those located within the solid.

When the shear wave velocity of the cylindrical solid inclusion is faster than the pressure wave velocity of the fluid (fast formation), the results again indicate a progressively slower flexural wave, as the ovality of the inclusion increases. The responses obtained at the receivers placed on the axis were weakly affected by the ovality ratio of the inclusion. Only the lower order modes were excited.

\section{Appendix A. Analytical 3D wave propagation solution for a solid circular cylinder submerged in a inviscid fluid medium}

Consider a cylindrical solid inclusion (Fig. 9) immersed in a spatially uniform fluid medium of infinite extent. Decomposing the homogeneous wave equations for elastic media in the usual way, by means of the now classical dilatational potential $\phi$ and shear potentials $\psi, \chi$, gives the three scalar wave equations in these potentials, with associated wave propagation velocities $\alpha, \beta$ and $\beta$, respectively. For a harmonic dilatational point source at an off-center position $\mathrm{O}$ in the fluid or solid, oscillating with a frequency $\omega$, the scalar wave equations lead to three Helmholtz equations, whose solution can be expressed in terms of the single dilatational potential for the incident waves, together with the set of potentials for scattered waves in both media.

\section{A.1. Incident field}

The incident dilatational potential is given by the expression

$$
\phi_{\text {inc }}=\frac{A \mathrm{e}^{\mathrm{i}\left(\omega / \alpha_{\mathrm{f}}\right)\left(\alpha_{\mathrm{f}} t-\sqrt{\left(x-x_{0}\right)^{2}+y^{2}+z^{2}}\right)}}{\sqrt{\left(x-x_{0}\right)^{2}+y^{2}+z^{2}}}
$$

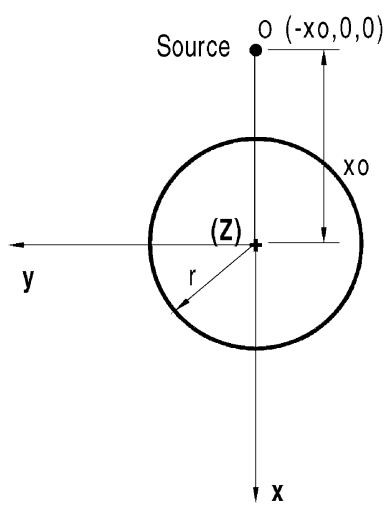

Fig. 9. Geometry of the problem for the analytical solution. 
in which the subscript 'inc' denotes the incident field, $A$ the wave amplitude, $\alpha_{\mathrm{f}}$ the acoustic (dilatational) wave velocity of the medium containing the source, and $i=\sqrt{-1}$.

Defining the effective wave numbers

$$
\begin{aligned}
& k_{\alpha}=\sqrt{\frac{\omega^{2}}{\alpha^{2}}-k_{z}^{2}}, \quad \operatorname{Im} k_{\alpha}<0, \quad k_{\beta}=\sqrt{\frac{\omega^{2}}{\beta^{2}}-k_{z}^{2}}, \quad \operatorname{Im} k_{\beta}<0, \\
& k_{\alpha_{\mathrm{f}}}=\sqrt{\frac{\omega^{2}}{\alpha_{\mathrm{f}}^{2}}-k_{z}^{2}}, \quad \operatorname{Im} k_{\alpha_{\mathrm{f}}}<0
\end{aligned}
$$

by means of the axial wavenumber $k_{z}$, the frequency of excitation $\omega$, and the wave velocities $\alpha, \beta$ and $\alpha_{\mathrm{f}}$, and Fourier transforming equation (A.1) in the $z$-direction, one obtains

$$
\phi_{\text {inc }}\left(\omega, x, y, k_{z}\right)=\frac{-\mathrm{i} A}{2} H_{0}^{(2)}\left(k_{\alpha_{\mathrm{f}}} \sqrt{\left(x-x_{0}\right)^{2}+y^{2}}\right)
$$

in which the $H_{n}^{(2)}(\ldots)$ are second Hankel functions of order $n$.

Eq. (A.3) expresses the incident field in terms of waves centered at the source point $O$, and not at the axis of the borehole. Graf's addition theorem is used to express the incident potential in terms of waves centered at the origin [44], leading to the expressions (in cylindrical coordinates)

$$
\begin{array}{ll}
\phi_{\text {inc }}\left(\omega, r, \theta, k_{z}\right)=-\frac{\mathrm{i} A}{2} \sum_{n=0}^{\infty}(-1)^{n} \varepsilon_{n} H_{n}^{(2)}\left(k_{\alpha_{\mathrm{f}}} r_{0}\right) J_{n}\left(k_{\alpha_{\mathrm{f}}} r\right) \cos n \theta & \text { when } r<r_{0} \\
\phi_{\text {inc }}\left(\omega, r, \theta, k_{z}\right)=-\frac{\mathrm{i} A}{2} \sum_{n=0}^{\infty}(-1)^{n} \varepsilon_{n} H_{n}^{(2)}\left(k_{\alpha_{\mathrm{f}}} r\right) J_{n}\left(k_{\alpha_{\mathrm{f}}} r_{0}\right) \cos n \theta & \text { when } r>r_{0}
\end{array}
$$

in which the $J_{n}(\ldots)$ are Bessel functions of order $n, \theta$ is the azimuth, and

$$
\varepsilon_{n}= \begin{cases}\frac{1}{2} & \text { if } n=0, \\ 1 & \text { if } n \neq 0\end{cases}
$$

where $r=\sqrt{x^{2}+y^{2}}$ is the distance to the receiver and $r_{0}$ the radial distance from the cylinder axis to the source

$$
\cos \theta=\frac{x}{r}, \quad \sin \theta=\frac{y}{r}
$$

\section{A.2. Scattered field in the fluid medium}

The scattered field in the fluid medium can be expressed, in the frequency-axial-wavenumber domain, using a form similar to that of the incident field, namely

$$
\phi_{\mathrm{sca}}^{\mathrm{f}}\left(\omega, r, \theta, k_{z}\right)=\sum_{n=0}^{\infty} A_{n} H_{n}^{(2)}\left(k_{\alpha_{\mathrm{f}}} r\right) \cos n \theta
$$

in which the subscript 'sca' denotes the scattered field, $A_{n}$ is an unknown coefficient to be determined from appropriate boundary conditions, the index 'f' identifies the fluid and $k_{\alpha_{\mathrm{f}}}=\sqrt{\left(\omega^{2} / \alpha_{\mathrm{f}}^{2}\right)-k_{z}}$. 


\section{A.3. Scattered field in the solid formation}

The scattered (or refracted) field in the solid circular formation, when the source is placed in the fluid medium, consists of standing waves, which can be expressed as

$$
\begin{aligned}
& \phi_{\mathrm{sca}}^{\mathrm{s}}\left(\omega, r, \theta, k_{z}\right)=\sum_{n=0}^{\infty} B_{n} J_{n}\left(k_{\alpha} r\right) \cos n \theta, \quad \psi_{\mathrm{sca}}^{\mathrm{s}}\left(\omega, r, \theta, k_{z}\right)=\sum_{n=0}^{\infty} C_{n} J_{n}\left(k_{\beta} r\right) \sin n \theta, \\
& \chi_{\mathrm{sca}}^{\mathrm{s}}\left(\omega, r, \theta, k_{z}\right)=\sum_{n=0}^{\infty} D_{n} J_{n}\left(k_{\beta} r\right) \cos n \theta
\end{aligned}
$$

in which index 's' identifies the solid region, and the coefficients $B_{n}, C_{n}$ and $D_{n}$ are unknown coefficients to be determined from the appropriate boundary conditions.

\section{A.4. Displacement field}

By imposing the continuity of displacements and stresses at the interface between the solid and the fluid mediums, namely $u_{r}^{\mathrm{s}}=u_{r}^{\mathrm{f}}, \sigma_{r r}^{\mathrm{s}}=\sigma_{r r}^{\mathrm{f}}$ and $\sigma_{r \theta}=\sigma_{r z}=0$, one obtains the unknown coefficients $A_{n}, B_{n}, C_{n}, D_{n}$ in Eqs. (A.6) and (A.7). Since an inviscid fluid was assumed, the tangential displacements at the boundary of the solid (i.e. $u_{\theta}, u_{z}$ ) may be different from those in the fluid (i.e. $u_{\theta}^{\mathrm{f}}, u_{z}^{\mathrm{f}}$ ). The unknown constants can be found by solving the system of the four stated boundary conditions for each summation index, $n$.

Having the constants, the scattered field can be calculated by means of the well-known equations relating potentials and displacements. Thus, the displacements can be achieved by applying partial derivatives to the potentials presented in Eqs. (A.6) and (A.7). After this procedure, the expressions for the scattered field in the solid and fluid are obtained:

$$
\begin{aligned}
& u_{r}\left(\omega, r, \theta, k_{z}\right)=\sum_{n=0}^{\infty} f_{n}(r) \cos n \theta, \quad u_{\theta}\left(\omega, r, \theta, k_{z}\right)=\sum_{n=0}^{\infty} g_{n}(r) \sin n \theta, \\
& u_{z}\left(\omega, r, \theta, k_{z}\right)=\sum_{n=0}^{\infty} h_{n}(r) \cos n \theta
\end{aligned}
$$

in which the functions $f_{n}, g_{n}$ and $h_{n}$ are given as in the following sections.

\section{A.4.1. Solid formation}

$$
\begin{aligned}
& f_{n}(r)=\left[\frac{n}{r} J_{n}\left(k_{\alpha} r\right)-k_{\alpha} J_{n+1}\left(k_{\alpha} r\right)\right] B_{n}+\frac{n}{r} J_{n}\left(k_{\beta} r\right) C_{n}-\mathrm{i} k_{z}\left[\frac{n}{r} J_{n}\left(k_{\beta} r\right)-k_{\beta} J_{n+1}\left(k_{\beta} r\right)\right] D_{n}, \\
& g_{n}(r)=-\frac{n}{r} J_{n}\left(k_{\alpha} r\right) B_{n}-\left[\frac{n}{r} J_{n}\left(k_{\beta} r\right)-k_{\beta} J_{n+1}\left(k_{\beta} r\right)\right] C_{n}+\mathrm{i} k_{z} \frac{n}{r} J_{n}\left(k_{\beta} r\right) D_{n}, \\
& h_{n}(r)=-\mathrm{i} k_{z} J_{n}\left(k_{\alpha} r\right) B n+k_{\beta}^{2} J_{n}\left(k_{\beta} r\right) D_{n}
\end{aligned}
$$

\section{A.4.2. Fluid medium}

$$
\begin{aligned}
f_{n}(r) & =\left[\frac{n}{r} H_{n}^{(2)}\left(k_{\alpha_{\mathrm{f}}} r\right)-k_{\alpha_{\mathrm{f}}} H_{n+1}^{(2)}\left(k_{\alpha_{\mathrm{f}}} r\right)\right] A_{n}, \quad g_{n}(r)=-\frac{n}{r} H_{n}^{(2)}\left(k_{\alpha_{\mathrm{f}}} r\right) A_{n}, \\
h_{n}(r) & =-\mathrm{i} k_{z} H_{n}^{(2)}\left(k_{\alpha_{\mathrm{f}}} r\right) A_{n}
\end{aligned}
$$

The total field in the $k_{z}$ wavenumber domain, for the medium where the source is placed (fluid medium), is calculated by adding the incident field obtained from Eqs. (A.4) or (A.5) by partial differentiation to the scattered field given in Eq. (A.10). 


\section{References}

[1] M.A. Biot, Propagation of elastic waves in cylindrical bore containing a fluid, J. Appl. Phys. 23 (1952) 997-1005.

[2] M. Schoenberg, Fluid and solid motion in the neighborhood of a fluid-filled borehole due to the passage of a low frequency elastic plane wave, Geophysics 51 (1986) 1191-1205.

[3] J.R. Lovell, B.E. Hornby, Borehole coupling at sonic frequencies, Geophysics 55 (1990) 806-814.

[4] C.H. Cheng, M.N. Toksöz, Elastic wave propagation in a fluid-filled borehole and synthetic acoustic logs, Geophysics 46 (1981) $1042-1053$.

[5] A.L. Kurkjian, S.-K. Chang, Acoustic multipole sources in fluid-filled boreholes, Geophysics 51 (1986) 148-163.

[6] A.F. Siggins, A.N. Stokes, Circumferential propagation of elastic waves on boreholes and cylindrical cavities, Geophysics 52 (1987) 514-529.

[7] K. Tezuka, C.H. Cheng, X.M. Tang, Modeling of low-frequency Stoneley-wave propagation in an irregular borehole, Geophysics 62 (1997) $1047-1058$.

[8] G.C. Herman, P.A. Milligan, Q. Dong, Analysis and removal of multiply scattered tube waves, Geophysics 65 (2000) $745-754$.

[9] C.H. Cheng, J. Zhang, D.R. Burns, Effects of in situ permeability on the propagation of Stoneley (tube) waves in a borehole, Geophysics 52 (1987) 1279-1289.

[10] L.J. Baker, G.A. Winbow, Multipole P-wave logging in formations altered by drilling, Geophysics 53 (1988) 1207-1218.

[11] A.K. Chan, L. Tsang, Propagation of acoustic waves in a fluid-filled borehole surrounded by a concentrically layered transversely isotropic formation, J. Acoust. Soc. Am. 74 (1983) 1605-1616.

[12] D.P. Schmitt, Acoustic multipole logging in transversely isotropic poroelastic formations, J. Acoust. Soc. Am. 86 (1989) $2397-2421$.

[13] K.J. Ellefsen, Elastic wave propagation along a borehole in an anisotropic medium, Ph.D. Thesis, MIT Press, Cambridge, MA, 1990.

[14] A.N. Norris, B.K. Sinha, Weak elastic anisotropy and the tube wave, Geophysics 58 (1983) 1091-1098.

[15] W. Dong, N.M. Toksöz, Borehole seismic-source radiation pattern in transversely isotropic media, Geophysics 60 (1995) $29-42$.

[16] C.T. Randall, Modes of noncircular fluid-filled boreholes in elastic formation, J. Acoust. Soc. Am. 89 (1991) $1002-1016$.

[17] A.L. Kurkjian, R.T. Coates, J.E. White, H. Schmidt, Finite-difference and frequency-wavenumber modeling of seismic monopole sources and receivers in fluid-filled boreholes, Geophysics 59 (1994) 1053-1064.

[18] C. Peng, J.M. Lee, N.M. Toksöz, Pressure in a fluid-filled borehole caused by a seismic source in stratified media, Geophysics 61 (1996) $43-55$.

[19] C.T. Randall, Multipole acoustic waveforms in non-axisymmetric boreholes and formations, J. Acoust. Soc. Am. 90 (1991) $1620-1631$.

[20] N. Cheng, C.H. Cheng, M.N. Toksöz, Borehole wave propagation in three dimensions, J. Acoust. Soc. Am. 97 (1995) $3483-3493$.

[21] L. Pochammer, Über die fortpflanzungsgeschwindigkeiten kleiner schwingungen in einem unbegrenzten isotropen kreiszylinder, J. Reine Angew. Math. 81 (1876) 324-336.

[22] V. Dayal, Longitudinal waves in homogeneous anisotropic cylindrical bars immersed in fluid, J. Acoust. Soc. Am. 93 (1993) $1249-1255$.

[23] K. Van den Abeele, O. Leroy, Complex harmonic wave scattering as the framework for investigation of bounded beam reflection and transmission at plane interfaces and its importance in the study of vibrational modes, J. Acoust. Soc. Am. 93 (1993) 308-323.

[24] J.M. Claeys, O. Leroy, Reflection and transmission of bounded sound beams on half-spaces and through plates, J. Acoust. Soc. Am. 72 (1982) 585-590.

[25] S. Furuyama, Y. Inoue, Weakly nonlinear waves and acoustic streaming produced by an oscillating rigid cylinder, J. Acoust. Soc. Am. 95 (1994) 708-717.

[26] T.K. Stanton, Sound scattering by rough elongated elastic objects. I. Means of scattered field, J. Acoust. Soc. Am. 92 (1992) $1641-1664$.

[27] S. Ramakrishna, P.R. Stepanishen, Acoustic scattering from cylinders with a plane of symmetry using internal multipole line source distributions, J. Acoust. Soc. Am. 93 (1993) 673-682.

[28] T.K. Stanton, P.H. Wiebe, D. Chu, Differences between sound scattering by weakly scattering spheres and finite-length cylinders with applications to sound scattering by zooplankton, J. Acoust. Soc. Am. 103 (1998) 254-264.

[29] D.V. Holliday, R.E. Pieper, Bioacoustical oceanography at high frequencies, ICES J. Mar. Sci. 52 (1995) $279-296$.

[30] Y.-S. Joo, J.-G. Ih, M.-S. Choi, Inherent background coefficients for acoustic resonance scattering from submerge, multilayered, cylindrical structures, J. Acoust. Soc. Am. 103 (1998) 900-910.

[31] G. Dahake, S.M. Gracewski, Finite difference predictions of P-SV wave propagation inside submerged solids. I. Liquid-solid interface conditions, J. Acoust. Soc. Am. 102 (1997) 2125-2137.

[32] G. Dahake, S.M. Gracewski, Finite difference predictions of P-SV wave propagation inside submerged solids. II. Effect of geometry, J. Acoust. Soc. Am. 102 (1997) 2138-2145.

[33] A. Niklasson, D. Subhendu, Scattering by an infinite transversely isotropic cylinder in a transversely isotropic medium, Wave Motion 27 (1998) 169-185.

[34] Y. Fan, A.N. Sinclair, F. Honarvar, Scattering of plane acoustic wave from a transversely isotropic cylinder encased in a solid elastic medium, J. Acoust. Soc. Am. 106 (1999) 1229-1236.

[35] A. Sarkissian, Radiation or scattering from multiple axisymmetric cylinders, J. Acoust. Soc. Am. 91 (1991) 3121-3125.

[36] M. Bouchon, A numerical simulation of the acoustic and elastic wavefields radiated by a source in a fluid-filled borehole embedded in a layered medium, Geophysics 58 (1993) 475-481. 
[37] W. Dong, M. Bouchon, M.N. Toksöz, Borehole seismic-source radiation in layered isotropic and anisotropic media: boundary element modeling, Geophysics 60 (1995) 735-747.

[38] M. Bouchon, K. Aki, Discrete wavenumber representation of seismic source wave fields, Bull. Seism. Soc. Am. 67 (1977) $259-277$.

[39] G.D. Manolis, D.E. Beskos, Boundary Element Methods in Elastodynamics Unwin Hyman (sold to Chapman \& Hall, London), 1988.

[40] D.E. Beskos, Boundary element methods in dynamic analysis: Part II (1986-1996), Appl. Mech. Rev. 50 (3) (1997) $149-197$.

[41] C.A. Brebbia, J.C.F. Telles, L.C. Wrobel, Boundary Element Technique, Springer, Berlin, 1984.

[42] A.J.B. Tadeu, E. Kausel, Green's functions for two-and-a-half dimensional elastodynamic problems, J. Eng. Mech. ASCE 126 (2000) 1093-1097.

[43] E. Kausel, Frequency domain analysis of undamped systems, J. Eng. Mech. ASCE 118 (1992) 121-734.

[44] G.N. Watson, A Treatise on the Theory of Bessel Functions, 2nd Edition, Cambridge University Press, Cambridge, 1980. 